\section{The Earth and its mountains}

SIR - I would like to comment on the diaskeuastic assessment of the relative merits of the three contending theories of mountainbuilding'. In my $1976^{2}$ and $1978^{3}$ papers, I pointed out that in relating the apparent secular accelerations of the Moon and Sun to the lunar and solar couples $N$ and $N^{\prime}$, the correct equation for the rate of change of angular momentum of the Earth is $(\mathrm{d} / \mathrm{d} t)$ $(\mathrm{C} \omega)=-N-N^{\prime}$, whereas in all six editions of his book The Earth, Sir Harold Jeffreys has adopted the form $C(\mathrm{~d} \omega \mathrm{d} t)=-N-$ $N^{\prime}$, which tacitly assumes the moment of inertia $C$ to be unchanging with time. This leads to quite unacceptable results if followed out straightforwardly, whereas Jeffreys handled the treatment in a way that obscures that the conclusions claimed do not in fact follow. Copies of these papers were duly sent to Sir Harold, but his only comment was, "Your references are out of date", which they were not, whence my 1980 paper $^{4}$. There can be no question of our being at loggerheads over it, for the day is long since past when there could be any dispute as to whether a dynamical equation is correct or not.

In his recent article ${ }^{5}$, Jeffreys states "that whether the Earth was hot or cold (initially) does not matter much for its history". But this is quite wrong, for if it started cool enough to be solid throughout, the known seismic data ${ }^{6}$ for the mantle prove that the initial radius would have been $370 \mathrm{~km}$ greater than the present radius of $6,371 \mathrm{~km}$. This is more than ten times the amount that thermal contraction could provide even when every factor is taken to maximize the latter. It implies a total reduction in surface area of about $50 \times 10^{9} \mathrm{~km}^{2}$, and a redisposal of more than $150 \times 10^{9}$ $\mathrm{km}^{3}$ of rock, as is shown in my recent book $^{7}$. This would be quite adequate for the more than 20 major periods of mountain-building literally unearthed by the geologists. Yet in a review article ${ }^{8}$ Jeffreys writes of the thermal-contraction hypothesis, "but no other suggested hypothesis even begins to explain it" (the needed contraction).

This hardly looks as if he "meant to refer to my 1965 paper"' omitted from both the 1970 and 1976 editions of The Earth, as he now says was his intention, but Jeffreys also omits any reference to my 1972 paper $^{9}$ wherein it is shown how all the terrestrial planets must have been formed initially solid throughout. The Moon and Mercury can hold no atmosphere now, and Mars barely so, and thus can never have passed through a gaseous or molten stage. Thermal contraction is therefore ruled out as an admissible hypothesis, and besides, where are the folded and thrusted mountains on Mars, Mercury and the Moon? (Volcanic mountains are not yet involved.) That there would be no such mountains on these bodies was predicted by the phase-change theory before any of the Mariner flights,

4. Lyttleton, R.A. Moon Planets 22, 13 (1980). and also that there would be no measurable dipole magnetic field on Mars, since the central pressure is far too low to produce the phase change.

On the identification of the core material with iron, the seismic data show that its uncompressed density (without change of state) would be only about $6 \mathrm{~g} \mathrm{~cm}^{-3}$, whereas iron and nickel, even when in liquid form, have densities 15 per cent and 30 per cent greater than this figure. Shockwave experiments have also been adduced to show that the core is of iron, but by arguments that are thermodynamically quite unsound. In the seismic case, infinitesimal waves and pressure differences are propagated through material already under high pressure and fairly high temperature, whereas with shock waves a severe impulsive shock moves into unstressed material at a normal temperature. The treatment history to be accorded to a volume element of material is totally different in the two cases, and chemical identification by comparison of velocity-density curves, which in fact fit very poorly if at all, is not possible. The alleged agreement with iron is rendered further suspect by the feature that, if the method were valid, the arguments would lead much more strongly to the conclusion that the mantle is made of aluminium. All this is explained in detail in another relevant but unreferenced paper ${ }^{10}$.

As for the vast verbal and pictorial literature of plate tectonics with its large number of purely asseverated assumptions, it may surprise some to learn that it simply fails to qualify as a scientific theory. I am sure Jeffreys fully agrees with this. Long ago, the great Poincare explained that "such descriptive accounts are not the role of physical theories, which should not introduce as many or more arbitrary constants (or verbal assumptions) as there are phenomena to be accounted for; they should establish connections between different experimental facts, and above all they should enable predictions to be made".

The real problem of orogeny is to account for over 20 major periods of mountain-building, and not just the one that produced the existing systems of folded and thrusted mountains. This the phasechange theory is able to do, as the mere calculation of the initial radius of an allsolid Earth alone makes abundantly clear.

\section{Institute of Astronomy,} R.A. LYTTLETON

\section{University of Cambridge,}

\section{Madingley Road,}

\section{Cambridge CB3 OHA, UK}

1. News and views Nature 300,681 (1982).

2. Lyttleton, R.A. Moon 16, 41 (1976)

3. Lyttleton, R.A. Moon Planets 18, 23 (1980).

5. Jeffreys, H. Geophys. J.R. astro. Soc. 71, 555 (1982)

6. Lyttleton, R.A. Proc. R.Soc. A 287, 471 (1965)

Lyttleton, R.A. The Earth and its Mountains, (Wiley, New York, 1982)

Jeffreys, H. A. Rev. Earth planet. Sci. 1, 11 (1973)

9. Lyttleton, R.A. Mon. Not. R. astr. Soc. 158, 463 (1972). 10. Moon 7, 442 (1973).

\section{Numerology puzzle}

SIR - Although numerology may be tempting, it does have some place in scientific work. An illuminating example arises in the theory of finite simple groups. The largest finite simple sporadic group has order approximately $10^{54}$ and is known as the Fisher-Griess "monster". Its smallest complex faithful linear representation has dimension 196,883 .

Two remarkable pieces of numerology stem from this group. First, the coefficient $q$ in the Fourier expansion

$$
\begin{gathered}
j(z)=q^{+}+744+196884 q+\ldots \\
(q=\exp (2 \pi \mathrm{i} z))
\end{gathered}
$$

of the classical elliptic modular function satisfies $196,884=196,883+1$ (see ref. 2 ) and second, the group is generated by a class of elements of order two (the Fischer involutions) such that the product of any two lies in one of nine conjugacy classes with the orders of the products given by the coefficients of the highest root as indicated in the accompanying $\mathrm{E}_{8}$ extended Dynkin diagram.

$1-2-3-i-5-\frac{\left.\right|^{3}-4}{6}-$

Neither of these coincidences is understood, although it has been shown that each conjugacy class of the "monster" is parametrized by a modular function whose coefficient of $q^{n}(\mathrm{n} \neq 0)$ is the character value afforded by a representation $H_{n}$ on an element in the class.

The numerological observations provide a numerical link between two initially unrelated areas: the recently developed theory of finite simple groups and, in the first case, the classical theory of modular functions, namely Lie theory, Kac-Moody theory and singularities. It is this critical contextual information which needs sifting if a coherent explanation for the numerology is to be found.

In the example cited in ref. 1 , we are presented with powers of $\pi$ but no suggested context. If one believes the evidence is strong enough to demand an explanation (and this is a matter of subjective probability), then one has to seek an appropriate mathematical context where such powers of $\pi$ occur. Perhaps the fundamental volumes of some classical groups furnish that context.

Finally, in dual-string models, the theory simplifies remarkably when working in a lorentzian space with signature $(25,1)$. This space occurs also in refs 3 and 4 and it is suggested that this numerology may be no coincidence, leading to some physical significance for the "monster" group.

Computer Science Department, JOHN MCKAY

\section{Concordia University,}

Montreal, Quebec, Canada H3G 1 M8

Maddox J. Nature 304, 11 (1983).

2. Thompson, J.G. Bull. Lond. Math. Soc. 11, 352-353 (1979).

3. Conway, J.H. J. Alg. 80, 159-163 (1983).

4. Conway, J.H. \& Sloane N.J.A., Bull. Amer. math. Soc. 6, 215-217 (1982). 